\title{
Voltage-Mode Lowpass, Bandpass and Notch Filters Using Three Plus-Type CCIIs
}

\author{
Jiun-Wei Horng, Zhao-Ren Wang, Chih-Cheng Liu \\ Department of Electronic Engineering, Chung Yuan Christian University, Chung-Li, Taiwan, China \\ E-mail:jwhorng@cycu.edu.tw \\ Received November 3, 2010; revised November 15, 2010; accepted November 18, 2010
}

\begin{abstract}
A single input and three outputs voltage-mode biquadratic filter using three plus-type second-generation current conveyors (CCII), two grounded capacitors and five resistors is presented. The proposed circuit offers the following features: realization of three filter functions, which are, lowpass, bandpass and notch filters, simultaneously, the use of only grounded capacitors. PSPICE simulation results are also included.
\end{abstract}

Keywords: Active Filter, Current Conveyor, Voltage-Mode, Biquad

\section{Introduction}

There is a growing interest in designing active filters using current conveyors. This is attributed to their high signal bandwidths, greater linearity and larger dynamic range $[1,2]$. The second-generation current conveyor (CCII) proves to be a versatile building block that can be used to implement a variety of high performance circuits, which are simple to construct. The current-feedback amplifier (CFA) exhibits the potentiality of extended operating bandwidth and relatively large value of slew rate compared to the conventional voltage-feedback amplifier. Note that a CFA is equivalent to a plus-type CCII with a voltage follower [3]. On the other hand, the use of only plus-type CCII in filters design has received considerable attention because of its simplicity [4-6].

The circuits consist of more filter functions mean more applications they can be used. Therefore, many cicuits with one input terminal and multiple output terminals were presented [7-11]. In 1994, Soliman [7] presented two CCII-based circuits that equivalent to the Kerwin-Huelsman-Newcomb biquad (KHN biquad) [8]. Both of these circuits use five CCIIs (three plus-type, two minus-type), six (or seven) resistors and two grounded capacitors to realize lowpass, bandpass and highpass transfer functions simultaneously as in the KHN circuit. In 1995, Senani and Singh [9] provided another approach to realize KHN biquad using CCIIs. The circuit uses five CCIIs (two plus-type, three minus-type), six grounded resistors and two grounded capacitors to obtain the lowpass, bandpass and highpass filter functions. In 1996, Soliman [10] presented several circuits that can realize lowpass, bandpass and highpass filters simultaneously using CFAs. In 1997, Horng et al. [11] proposed a lowpass, bandpass and highpass filter uses four plus-type CCIIs and seven passive components. Recently, several KHN biquads using various active elements were proposed in [12-15]. However, the notch filter cannot be obtained without additional circuitry from these circuit configurations [7-15]. In 1994, [16] Chang proposed a lowpass, bandpass and notch filter using three CFAs, three resistors and two grounded capacitors.

In this paper, we proposed a voltage-mode biquadratic filter with single input and three outputs using three plus-type CCIIs, two grounded capacitors and five resistors. Lowpass, bandpass and notch filters can be obtained simultaneously from the same circuit configuration. With respect to the previous CFA based lowpass, bandpass and notch biquadratic filter circuit in [16], the proposed circuit employs simpler active components (plus-type CCIIs).

\section{Circuit Description}

Using standard notation, the port relations of an ideal plus-type CCII can be characterized by

$$
\left[\begin{array}{l}
i_{y} \\
v_{x} \\
i_{z}
\end{array}\right]=\left[\begin{array}{lll}
0 & 0 & 0 \\
1 & 0 & 0 \\
0 & 1 & 0
\end{array}\right]\left[\begin{array}{l}
v_{y} \\
i_{x} \\
v_{z}
\end{array}\right]
$$


The proposed configuration is shown in Figure 1. Assuming $R_{3}=R_{4}=R_{5}=R$, The transfer functions can be expressed as

$$
\begin{aligned}
& \frac{V_{\text {out } 1}}{V_{\text {in }}}=\frac{\frac{G_{1} G^{2}}{s^{2}+s \frac{G_{2}\left(G_{1}+G-G_{2}\right)}{C_{1}\left(G_{1}+G-G_{2}\right)}+\frac{G^{2}}{C_{1} C_{2}}}}{\text {. }} \\
& \frac{V_{\text {out } 2}}{V_{\text {in }}}=\frac{-s \frac{G_{1} G}{C_{1}\left(G_{1}+G-G_{2}\right)}}{s^{2}+s \frac{G^{2}}{C_{1}\left(G_{1}+G-G_{2}\right)}+\frac{G^{2}}{C_{1} C_{2}}} \\
& \frac{V_{\text {out } 3}}{V_{\text {in }}}=\frac{s^{2} \frac{G_{1}}{G_{1}+G-G_{2}}+\frac{G_{1} G^{2}}{C_{1} C_{2}\left(G_{1}+G-G_{2}\right)}}{s^{2}+s \frac{G^{2}}{C_{1}\left(G_{1}+G-G_{2}\right)}+\frac{G^{2}}{C_{1} C_{2}}}
\end{aligned}
$$

From (2)-(4) it can be seen that a lowpass response is obtained from $V_{\text {out } 1}$, a bandpass response is obtained from $V_{\text {out } 2}$ and a notch response is obtained from $V_{\text {out } 3}$. The proposed circuit uses three plus-type CCIIs, two grounded capacitors and five resistors. The design of using only grounded capacitors is attractive, because grounded capacitor can be implemented on a smaller area than the floating counterpart and it can absorb equivalent shunt capacitive parasitics [17-19]. In all cases the resonance angular frequency $\omega_{o}$ and the quality factor $Q$ are given by

$$
\omega_{o}=G \sqrt{\frac{1}{C_{1} C_{2}}}
$$

and

$$
Q=\frac{G_{1}+G-G_{2}}{G} \sqrt{\frac{C_{1}}{C_{2}}}
$$

The resonance angular frequency can be controlled by $G$. The quality factor can be orthogonally controlled by $G_{1}$ or $G_{2}$.

\section{Sensitivity Analysis}

Taking into account the non-idealities of the plus-type

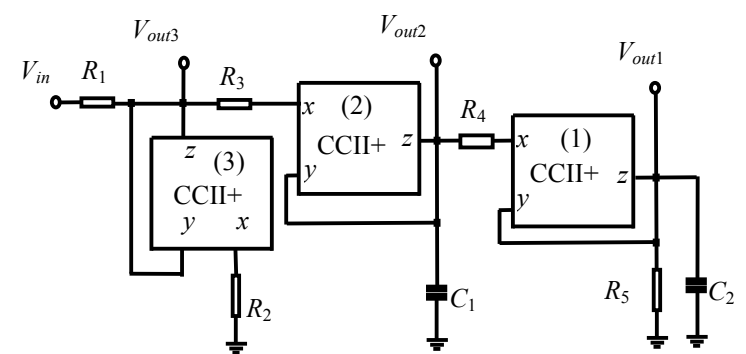

Figure 1. The proposed voltage-mode biquadratic filter.
CCII, the characteristics of the non-ideal CCII can be given by $i_{y}=0, i_{z}=\alpha(s) i_{x}$ and $v_{x}=\beta(s) v_{y}$, where $\alpha(s)$ and $\beta(s)$ represent the frequency transfers of the internal current and voltage followers of the plus-type CCII, respectively. They can be approximated by the following first order lowpass functions [20].

$$
\begin{aligned}
& \alpha(s)=\frac{\alpha_{o}}{1+s / \omega_{\alpha}} \\
& \beta(s)=\frac{\beta_{o}}{1+s / \omega_{\beta}}
\end{aligned}
$$

where $\alpha_{o}=0.9914, \omega_{\alpha}=3.8 \times 10^{9} \mathrm{rad} / \mathrm{s}, \quad \beta_{o}=0.9999$, $\omega_{\beta}=6.48 \times 10^{9} \mathrm{rad} / \mathrm{s}$. Assuming the circuits are working at frequencies much lower than the corner frequencies of $\alpha(s)$ and $\beta(s)$, namely, $\alpha(s)=\alpha=1-\varepsilon_{1}$ and $\varepsilon_{1}$ $\left(\left|\varepsilon_{1}\right|<<1\right)$ denotes the current tracking error and $\beta(s)=\beta=1-\varepsilon_{2}$ and $\varepsilon_{2} \quad\left(\left|\varepsilon_{2}\right|<<1\right)$ denotes the voltage tracking error of the plus-type CCII. The denominator of the non-ideal output voltage function for Figure 1 is

$$
D(s) \cong s^{2}+s \frac{G^{2} \alpha_{2} \beta_{2}}{C_{1}\left(G_{1}+G-G_{2} \alpha_{3} \beta_{3}\right)}+\frac{G^{2} \alpha_{1} \beta_{1}}{C_{1} C_{2}}
$$

The resonance angular frequency $\omega_{o}$ and quality factor $Q$ are obtained by

$$
\omega_{o}=G \sqrt{\frac{\alpha_{1} \beta_{1}}{C_{1} C_{2}}}
$$

and

$$
Q=\frac{G_{1}+G-G_{2} \alpha_{3} \beta_{3}}{G \alpha_{2} \beta_{2}} \sqrt{\frac{C_{1} \alpha_{1} \beta_{1}}{C_{2}}}
$$

Because of the tolerances in component values and the non-idealities of the plus-type CCIIs, the response of the actual assembled filter will deviate from the ideal response. As a means for predicting such deviations, the filter designer employs the concept of sensitivity. The sensitivity function is defined as:

$$
S_{x}^{y}=\frac{\partial y}{\partial x} \frac{x}{y}
$$

The active and passive sensitivities of $\omega_{o}$ and $Q$ of the proposed filter are:

$$
\begin{gathered}
S_{G}^{\omega_{o}} \cong 1, \quad-S_{C_{1}, C_{2}}^{\omega_{o}} \cong S_{\alpha_{1}, \beta_{1}}^{\omega_{o}} \cong \frac{1}{2}, S_{G}^{Q} \cong \frac{-G_{1}+G_{2}}{G+G_{1}-G_{2}}, \\
S_{G_{1}}^{Q} \cong \frac{G_{1}}{G+G_{1}-G_{2}}, \quad S_{G_{2}}^{Q} \cong \frac{-G_{2}}{G+G_{1}-G_{2}}, \\
S_{C_{1}}^{Q} \cong-S_{C_{2}}^{Q} \cong S_{\alpha_{1}, \beta_{1}}^{Q} \cong \frac{1}{2}, \quad S_{\alpha_{3}, \beta_{3}}^{Q} \cong \frac{-G_{2}}{G+G_{1}-G_{2}} .
\end{gathered}
$$


These values have been calculated by assuming that $\alpha_{1}, \alpha_{2}, \alpha_{3}, \beta_{1}, \beta_{2}$ and $\beta_{3}$ are near unity.

\section{Simulation Results}

The proposed circuit was simulated using PSPICE. The plus-type CCII was implemented using one AD844. The supply voltages are chosen as $\pm 12 \mathrm{~V}$. The following setting were selected to obtain the lowpass, bandpass and notch filters: $R_{1}=R_{2}=R_{3}=R_{4}=R_{5}=1 \mathrm{k} \Omega, C_{1}=C_{2}=1 \mathrm{nF}$ with $Q=1$ and $f_{o}=159.15 \mathrm{KHz}$. Figures 2 (a), (b) and (c) represent the simulated frequency responses for the lowpass $\left(V_{\text {out } 1}\right)$, bandpass $\left(V_{\text {out } 2}\right)$ and notch $\left(V_{\text {out } 3}\right)$ filters of Figure 1, respectively. The simulation results are coherent with the theoretical analyses. The CCII has parasitic resistor from the $z$ terminal to the ground $\left(R_{z}[21]\right)$. When the $z$ terminal load of the CCII is a capacitor $(C)$, it in troduces a pole produced by $R_{z}$ and $C$ at low frequency. This explains why Figure 2(b) has non-ideal phase responses at low frequencies. This effect can be minimized by using larger loading capacitor or operating the filter in high frequencies.

\section{Conclusions}

In this paper, a new single input and three outputs voltage-mode biquadratic filter is presented. The proposed circuit uses three plus-type CCIIs, two grounded capaci-

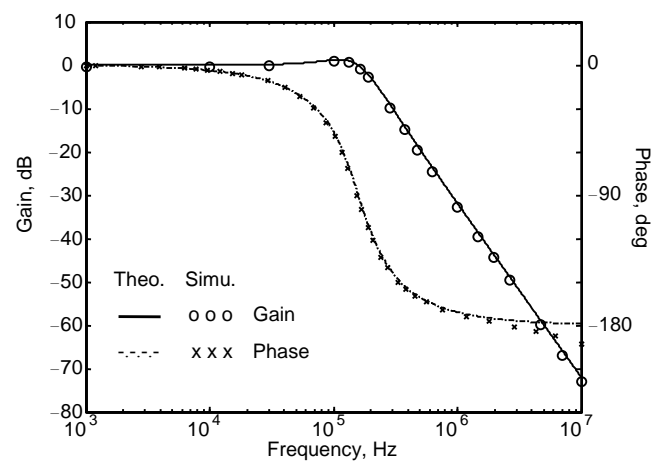

(a)

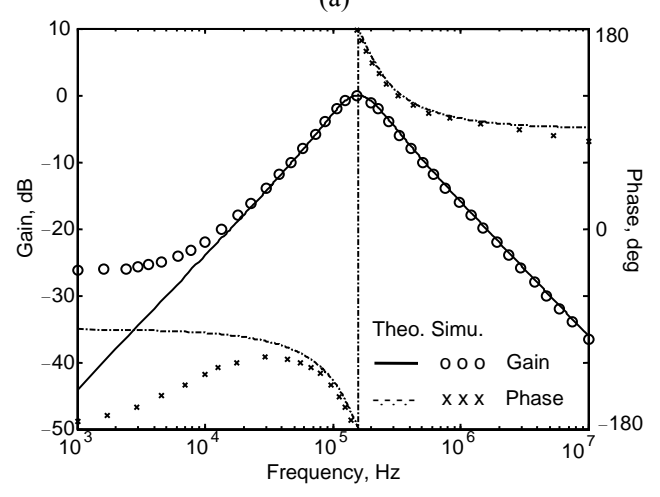

(b)

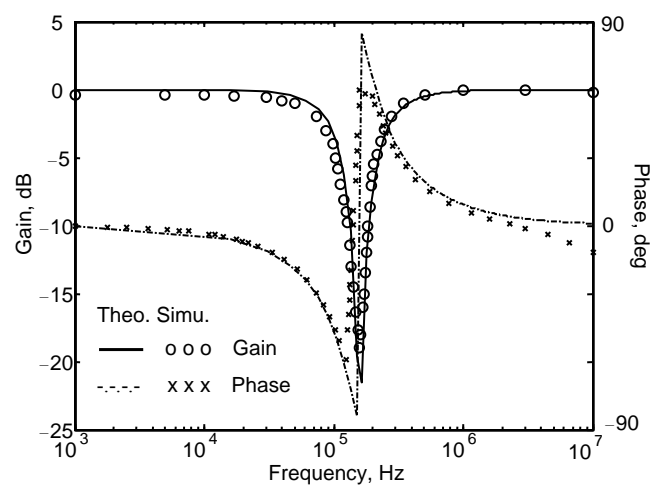

(c)

Figure 2. Simulated frequency responses of Figure 1 design with $C_{1}=C_{2}=1 \mathrm{nF}$ and $R_{1}=R_{2}=R_{3}=R_{4}=R_{5}=1 \mathrm{k} \Omega$. (a) lowpass filter $\left(V_{\text {out } 1}\right)$; (b) bandpass filter $\left(V_{\text {out } 2}\right)$, (c) notch filter $\left(V_{\text {out3 }}\right)$.

tors and five resistors. The new circuit offers several advantages, such as the realization of lowpass, bandpass, and notch filter functions, simultaneously, in the same circuit configuration; the use of only three plus-type CC -IIs; orthogonally controllable of resonance angular frequency and quality factor and the use of only grounded capacitors.

\section{References}

[1] C. Toumazou, F. J. Lidgey and D. G. Haigh, "Analogue IC Design: The Current-mode Approach," Peter Peregrinus Ltd., London, 1990.

[2] G. W. Roberts and A. S. Sedra, "All Current-Mode Frequency Selective Circuits," Electronics Letters, Vol. 25, No. 12, 1989, pp. 759-761. doi:10.1049/el:19890513

[3] J. A. Svoboda, L. McGory and S. Webb, "Applications of a Commercially Available Current Conveyor," International Journal of Electronics, Vol. 70, No. 1, 1991, pp. 159-164. doi:10.1080/00207219108921266

[4] M. Higashimura and Y. Fukui, "Universal Filter Using Plus-Type CCIIs," Electronics Letters, Vol. 32, No. 9, 1996, pp. 810-811. doi:10.1049/el:19960518

[5] T. Tsukutani, Y. Sumi and N. Yabuki, "Versatile Current-Mode Biquadratic Circuit Using Only Plus Type CCCIIs and Grounded Capacitors," International Journal of Electronics, Vol. 94, No. 12, 2007, pp. 1147-1156. doi:10.1080/00207210701789998

[6] J. W. Horng, "Voltage/Current-Mode Universal Biquadratic Filter Using Single CCII+," Indian Journal of Pure \& Applied Physics, Vol. 48, No. 10, 2010, pp. 749-756.

[7] A. M. Soliman, "Kerwin-Huelsman-Newcomb Circuit using Current Conveyors," Electronics Letters, Vol. 30, No. 24, 1994, pp. 2019-2020. doi:10.1049/el:19941368

[8] W. Kerwin, L. Huelsman and R. Newcomb, "State Variable Synthesis for Insensitive Integrated Circuit Transfer Functions," IEEE Journal of Solid State Circuits, Vol. 2, No. 3, 1967, pp. 87-92. doi:10.1109/JSSC.1967.1049798 
[9] R. Senani and V. K. Singh, "KHN-Equivalent Biquad Using Current Conveyors," Electronics Letters, Vol. 31, No. 8, 1995, pp. 626-628. doi:10.1049/el:19950422

[10] A. M. Soliman, "Applications of the Current Feedback Amplifiers," Analog Integrated Circuits and Signal Processing, Vol. 11, 1996, pp. 265-302. doi:10.1007/BF002 40490

[11] J. W. Horng, J. R. Lay, C. W. Chang and M. H. Lee, "High Input Impedance Voltage-Mode Multifunction Filters Using Plus-Type CCIIs," Electronics Letters, Vol. 33, No. 6, 1997, pp. 472-473. doi:10.1049/el:19970297

[12] E. Altuntas and A. Toker, "Realization of Voltage and Current Mode KHN Biquads Using CCCIIs," AEU International Journal of Electronics and Communications, Vol. 56, No. 1, 2002, pp. 45-49. doi:10.1078/1434-841154100071

[13] A. M. Soliman, "Kerwin Huelsman Newcomb Filter Using Inverting CCII," Journal of Active and Passive Electronic Devices, Vol. 3, 2008, pp. 273-279.

[14] S. Minaei and M. A. Ibrahim, "A Mixed-Mode KHNBiquad Using DVCC and Grounded Passive Elements Suitable for Direct Cascading," International Journal of Circuit Theory and Applications, Vol. 37, 2009, pp. 793810. doi:10.1002/cta.493

[15] J. Koton, N. Herencsar and K. Vrba, "Single-Input ThreeOutput Variable Q and $\omega_{0}$ Filters Using Universal Voltage Conveyors," International Journal of Electronics, Vol. 97, No. 5, 2010, pp. 531-538. doi:10.1080/0020721 0903486864
[16] C. M. Chang, C. S. Hwang and S. H. Tu, "Voltage-Mode Notch, Lowpass and Bandpass Filter Using Current-FeedBack Amplifiers," Electronics Letters, Vol. 30, No. 24 , 1994, pp. 2022-2023. doi:10.1049/el:19941416

[17] M. Bhushan and R. W. Newcomb, "Grounding of Capacitors in Integrated Circuits," Electronic Letters, Vol. 3, No. 4, 1967, pp. 148-149. doi:10.1049/el:19670114

[18] E. Yuce and S. Minaei, "ICCII-Based Universal Current-Mode Analog Filter Employing Only Grounded Passive Components," Analog Integrated Circuits and Signal Processing, Vol. 58, 2009, pp. 161-169. doi:10.1007/ s10470-008-9225-2

[19] C. M. Chang, A. M. Soliman and M. N. S. Swamy, "Analytical Synthesis of Low-Sensitivities High-Order Voltage-Mode DDCC and FDCCII-Grounded R and C All-Pass Filter Structures," IEEE Transactions on Circuits and Systems I: Regular Papers, Vol. 54, No. 7, 2007, pp. 1430-1443. doi:10.1109/TCSI.2007.900183

[20] A. Fabre, O. Saaid and H. Barthelemy, "On the Frequency Limitations of the Circuits Based on Second Generation Current Conveyors," Analog Integrated Circuits and Signal Processing, Vol. 7, 1995, pp. 113-129. doi:10.100 7/BF01239166

[21] J. W. Horng, "High-Order Current-Mode and Transimpedance-Mode Universal Filters with Multiple-Inputs and Two-Outputs Using MOCCIIs," Radioengineering, Vol. 18, No. 4, 2009, pp. 537-543. 